\title{
Investigating Communications Energy Efficiency Tradeoff Between UAV Users and Small-cell Users
}

\author{
Ramin Hashemi ${ }^{\dagger}$, Mohammad Robat Mili ${ }^{\ddagger}$, Samad Ali ${ }^{\dagger}$, Hamzeh Beyranvand*, and Matti Latva-aho ${ }^{\dagger}$ \\ ${ }^{\dagger}$ Centre for Wireless Communications (CWC), University of Oulu, Oulu, Finland, \\ Emails: \{ramin.hashemi, samad.ali, matti.latva-aho\}@oulu.fi \\ Islamic Azad University, Tehran, Iran, \\ Email: mohammad.robatmili@ieee.org \\ *Department of Electrical Engineering, Amirkabir University of Technology (Tehran Polytechnic), Tehran, Iran, \\ Email: beyranvand@aut.ac.ir
}

\begin{abstract}
In this paper, a novel method is proposed to study the tradeoff between energy efficiency (EE) of small-cell users and unmanned aerial vehicles (UAV) users in multi-cell orthogonal frequency division multiple access (OFDMA)-based networks. Contrary to the prior works that only maximize the EE of the UAV network subject to some constraints on transmit power of UAV users, we formulate a multi-objective optimization problem (MOOP) that jointly maximize the EE of small-cell and UAV users while guaranteeing the minimum rate for UAV users as well as maximum transmit powers for the corresponding small-cell and UAV BSs. The proposed MOOP is transformed into a single optimization problem (SOOP) by the weighted Tchebycheff approach. Then, an iterative technique is used to optimize alternatively subchannels and transmission powers of small-cell and UAV networks at each step. Numerical results show that a substantial performance gain can be obtained over the existing solutions.
\end{abstract}

Index Terms: Energy efficiency, heterogeneous network, multiobjective optimization problem, OFDMA, small-cell, unmanned aerial vehicles.

\section{INTRODUCTION}

The historical evolution of unmanned aerial vehicles (UAV) [1] as a flying base station (BS) has become a novel paradigm to improve the total coverage and capacity of the terrestrial networks. They can establish a Line-of-sight (LoS) channel between the ground users and aerial UAV BS and can be easily deployed during a system failure. Therefore, the use of aerial UAV base stations can boost the quality of service (QoS) of the network in terms of higher data rate, higher reliability, etc. There are two general categories of UAV deployments, rotarywing, and fixed-wing. The fixed-wing UAVs have high speed and large payloads, while, rotary-wing UAVs have limited payload and can move to any direction in a stationary manner [2].

In $5 \mathrm{G}$ radio access networks (RAN) ultra-dense deployment of small-cells (SC) can improve the areal spectral efficiency of broadband access networks (BAN) [3]. In heterogeneous RANs, referred to as HetNets, SCs are connected to the core network through macro base stations (MBSs) in a wireless backhaul or directly by a gateway such as fiber optics communication. Although the densification technique improves areal transmission capacity, however, it raises the so-called backhauling bottleneck especially in MBSs [4]. Therefore, the UAV base stations can be deployed concurrently together with SC networks where the SCs are connected to the heterogeneous radio access networks (RAN) through a gateway. In this concept, new challenges are introduced including the impact of SC users on the performance of UAV users and vice versa. Hence, investigating the performance of jointly deployed UAV and SC networks in terms of energy efficiency (EE) and achievable data rate is of paramount importance.

The work in [5] investigates the fundamental performance analysis of the UAV communication with underlaid device-todevice (D2D) communication links where the coverage probability for D2D and cellular users are derived for two scenarios, i.e. static and mobile aerial UAV BS. In [6] the role of a UAVenabled network is studied in public safety communications in case of emergencies like an earthquake. The authors in [7] investigate the performance of heterogeneous RANs where the UAV base stations act as the relay node between MBSs and SCs. However, none of the prior works have studied the coexistence of UAV and SC networks jointly.

In conventional system designs, generally, the most noticeable performance metric is selected as the optimization objective and the remaining performance metrics are considered as the constraints of the optimization problem. Such a single objective optimization problem (SOOP) approaches may give unfair and unreasonable adjustments in real applications because of over-emphasizing the importance of one metric to the rest. Particularly, in small-cell UAV networks (SUAVN), the sum rate or the EE of UAV users may be chosen as optimization objective while small-cell transmit power and its interference on UAV users are treated as the constraint. Hence, more realistic optimization should be considered to simultaneously satisfy multiple objectives. Accordingly, the multi-objective optimization problem (MOOP) can be adjusted for solving the problems with more realistic scenarios [8]. Recent works have been studied using MOOP in wireless systems design [9]-[12]. In [9], a MOOP has been formulated to optimize simultaneously the data rates and harvested powers in 
multi-user multiple-input multiple-output (MIMO) broadcast networks while designing simultaneous wireless information and power transfer. A MOOP that jointly maximizes the ergodic capacity and minimizes the average transmission power of the secondary users is formulated in [10] and solved by transferring it into a SOOP by using the $\varepsilon$-constraint method. Three crucial issues on resource management including powerefficient improvement, user-fairness guarantee, and non-ideal channel reciprocity effect mitigation have been addressed jointly using the framework of MOOP in [11].

To the best of our knowledge, there is no reported work in the literature that directly focuses on the tradeoff between the EE of the UAV network and the EE of the small-cell communication links. This motivates us to formulate a MOOP framework that jointly maximizes the EE of UAV and smallcell users, enabling us to investigate a tradeoff between the achievable EEs of SUAVN in downlink orthogonal frequencydivision multiple access (OFDMA) system. Towards solving the formulated MOOP, we first apply a weighted Tchebycheff method and then propose an iterative approach that alternatively assigns subchannels to UAV and small-cell users and allocates power to UAV aerial base stations and smallcells, respectively. To demonstrate the advantage of MOOP formulation, we consider a SOOP as a special case of MOOP where the EE of only small-cell users is maximized subject to the constraints on the minimum data rate of each UAV user and the total transmit power of the small-cell base stations. Numerical results reveal a substantial performance gain of the MOOP over conventional SOOP where the EE of only smallcell users is maximized. Thus, MOOP formulation in SUAVNs can show the whole capability of network resources.

The rest of the paper is organized as follows. Section II presents the considered system model architecture. In Section III, we formulate the EE MOOP framework of subchannel assignment and power control in the UAV-SC network and propose the sequential quadratic algorithm to solve the presented non-convex problem. In Section IV, numerical results are reported to evaluate the proposed algorithm and study the tradeoff between the two objectives. Finally, the paper is concluded in Section V.

\section{SYSTEM MODEL}

Consider the downlink of an OFDMA-based small-cell UAV network consisting of set $\mathcal{C}$ of $\mathrm{C}$ cells with one SC BS and one UAV BS at each cell. $m_{c}$ and $k_{c}$ users are served by UAV base station (UAV-BS) and SC base station (SC-BS), respectively. The UAV base station $c$ serves $m_{c}$ UAV users (U-UEs) and the SC at cell $c$ is associated with $k_{c}$ small-cell UE (S-UEs). Hence, the total number of U-UEs and S-UEs in the entire network are $M=\sum_{c=1}^{C} m_{c}$ and $K=\sum_{c=1}^{C} k_{c}$, respectively. The bandwidth of $W \mathrm{~Hz}$ is divided into $N$ subchannels such that each subchannel with the bandwidth of $W_{c}=W / N \mathrm{~Hz}$. The subchannels are modeled as block flat-fading channels. In subchannel $n$, time slot $t$ and cell $c$, we denote the instantaneous channel power gains for the link between the $c$ th SBS and the $k$ th SU in that SBS by $h_{k, c}^{n}(t)$

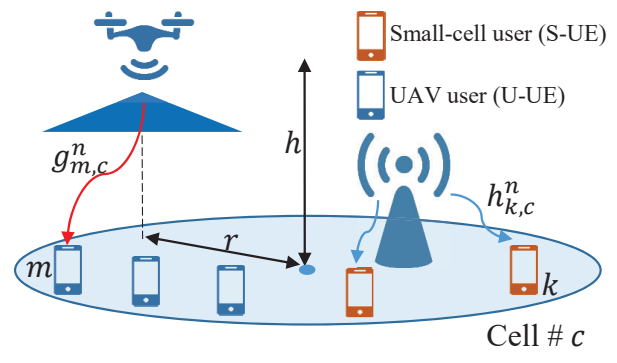

Fig. 1: The network model (the subchannel index $n$ is considered as an example).

and the link between the $c$ th UAV BS and the $m$ th U-UE as $g_{m, c}^{n}(t)$. In the cell $c$, the power allocated to the S-UE $k$ in the subchannel $n$ is denoted by $p_{k, c}^{n}$. The power allocated to the U-UE $m$ by the BS $c$ on the subchannel $n$ is given by $\hat{p}_{m, c}^{n}$. The noise power is given by $N_{0}$. It is also assumed that a subchannel at each cell is allocated only to one user. Fig. 1, presents an illustration of the system model.

The instantaneous received signal-to-interference-plus-noise ratio (SINR) at the S-UE $k$ in $\mathrm{BS} c$ on subchannel $n$ can be written as

$$
\Gamma_{k, c}^{n, \mathrm{~S} \text {-UE }}=\frac{p_{k, c}^{n} h_{k, c}^{n}}{N_{0}+\sum_{\substack{q=1 \\ q \neq c}}^{C} \sum_{m=1}^{m_{c}} \hat{p}_{m, q}^{n} g_{k, q}^{\prime n}+\sum_{\substack{i=1 \\ i \neq c}}^{C} \sum_{j=1}^{k_{i}} p_{j, i}^{n} h_{k, i}^{\prime n}},
$$

where $g_{k, q}^{\prime n}$ and $h_{k, i}^{\prime n}$ are the instantaneous channel power gains of the channels between the other UAV BSs or SCs and the S-UE $k$ at subchannel $n$, respectively. In addition, the instantaneous received SINR at the U-UE $m$ in subchannel $n$ is given by

$$
\Gamma_{m, c}^{n, \mathrm{U}-\mathrm{UE}}=\frac{\hat{p}_{m, c}^{n} g_{m, c}^{n}}{N_{0}+\sum_{\substack{j=1 \\ j \neq c}}^{C} \sum_{k=1}^{k_{c}} p_{k, j}^{n} \hat{h}_{m, j}^{\prime n}+\sum_{\substack{i=1 \\ i \neq c}}^{C} \sum_{q=1}^{m_{i}} \hat{p}_{q, i}^{n} \hat{g}_{m, i}^{\prime n}}
$$

where $\hat{h}_{m, j}^{\prime n}$ and $\hat{g}_{m, i}^{\prime n}$ are the instantaneous power gains of the channel between the other UAV BSs or SCs and U-UE $m$ in cell $c$ at subchannel $n$, respectively.

To model the air to ground channel between UAV and users, LoS and none-LoS (NLoS) components with probabilities $P_{\mathrm{LoS}}$ and $P_{\mathrm{NLoS}}$, respectively [5] are considered. Moreover, the impact of small-scale fading can be ignored in this case, and finally we have

$$
g_{m, c}^{n}= \begin{cases}\left|d_{m, c}\right|^{-\kappa}, & \text { with probability } P_{\mathrm{LoS}}, \\ \eta\left|d_{m, c}\right|^{-\kappa}, & \text { with probability } P_{\mathrm{NLoS}}\end{cases}
$$

where $\kappa$ shows the path loss exponent and $\eta$ is an excess attenuation factor due to the NLoS connection caused by the shadowing effect and $d_{m, c}$ indicates the distance between UAV BS at cell $c$ and user $m$. To evaluate $P_{\mathrm{LoS}}$ we use

$$
P_{\mathrm{LoS}}=\frac{1}{1+\kappa_{2} \exp \left(-\kappa_{1}\left(\theta-\kappa_{2}\right)\right)}
$$


where $\kappa_{1}$ and $\kappa_{2}$ depend on the environmental factors [16] and $\theta=\frac{180}{\pi} \sin ^{-1}\left(\frac{h}{\sqrt{h^{2}+r^{2}}}\right), P_{\mathrm{LoS}}=1-P_{\mathrm{NLoS}}$. Note that $g_{k, q}^{\prime n}$ and $\hat{g}_{m, i}^{\prime}$ in (1) and (2) follow the same channel model presented here. In the following section, a MOOP formulation is offered to present a tradeoff between the EE of U-UEs and S-UEs.

\section{MOOP FORMULATION FOR ENERGY EFFICIENCIES}

In this section, to obtain a novel tradeoff between the EE of U-UEs and S-UEs, we formulate a MOOP that jointly maximizes the EE of S-UEs $\left(\eta_{\mathrm{EE}_{\mathrm{S}-\mathrm{UE}}}\right)$ and the EE of UAV users $\left(\eta_{\mathrm{EE}_{\mathrm{U}-\mathrm{UE}}}\right)$ as

$$
\begin{array}{ll}
\underset{\{\mathbf{p}, \hat{\mathbf{p}}, \boldsymbol{\varphi}, \boldsymbol{\psi}\}}{\max } \eta_{\{\mathbf{p}, \hat{\mathbf{p}}, \boldsymbol{\varphi}, \boldsymbol{\psi}\}} \eta_{\mathrm{EE}_{\mathrm{U}-\mathrm{UE}}}, \\
\text { s.t. } \quad \sum_{k=1}^{k_{c}} \sum_{n=1}^{N} p_{k, c}^{n} \leq P_{\max , c}^{\mathrm{SC}} \forall c \in \mathcal{C}, \\
\quad \sum_{m=1}^{m_{c}} \sum_{n=1}^{N} \hat{p}_{m, c}^{n} \leq P_{\max , c}^{\mathrm{UAV}} \forall c \in \mathcal{C}, \\
\quad \sum_{n=1}^{N} \psi_{m, c}^{n} \ln \left(1+\Gamma_{m, c}^{n, \mathrm{U}-\mathrm{UE}}\right) \geq R_{m, c}^{\min }, \\
\quad \sum_{k=1}^{k_{c}} \varphi_{k, c}^{n} \leq 1, \forall n, \forall c, \varphi_{k, c}^{n} \in\{0,1\}, \forall n, k, c \\
\quad \sum_{m=1}^{m_{c}} \psi_{m, c}^{n} \leq 1, \forall n, \forall c, \psi_{m, c}^{n} \in\{0,1\}, \forall n, m, c
\end{array}
$$

where

$$
\begin{aligned}
& \eta_{\mathrm{EE}_{\mathrm{S}-\mathrm{UE}}}= \frac{\sum_{c=1}^{C} \sum_{k=1}^{k_{c}} \sum_{n=1}^{N} \varphi_{k, c}^{n} \ln \left(1+\Gamma_{k, c}^{n, \mathrm{~S}-\mathrm{UE}}\right)}{\sum_{c=1}^{C} \sum_{k=1}^{k_{c}} \sum_{n=1}^{N} p_{k, c}^{n}+\sum_{c=1}^{C} p_{c_{c}}}, \\
& \eta_{\mathrm{EE}_{\mathrm{U}-\mathrm{UE}}}=\frac{\sum_{c=1}^{C} \sum_{m=1}^{m_{c}} \sum_{n=1}^{N} \psi_{m, c}^{n} \ln \left(1+\Gamma_{m, c}^{n, \mathrm{U}-\mathrm{UE}}\right)}{\sum_{c=1}^{C} \sum_{m=1}^{m_{c}} \sum_{n=1}^{N} \hat{p}_{m, c}^{n}+\sum_{c=1}^{C} \hat{p}_{c_{c}}} .
\end{aligned}
$$

In (5), $\varphi_{k, c}^{n}$ is a binary variable for subchannel allocation in small-cell network such that $\varphi_{k, c}^{n}=1$ if subchannel $n$ is allocated to S-UE $k$ at cell $c$ and $\varphi_{k, c}^{n}=0$, otherwise. Likewise, $\psi_{m, c}^{n}$ is a binary variable for subchannel allocation in UAV network such that $\psi_{m, c}^{n}=1$ if subchannel $n$ is allocated to U-UE $m$ at cell $c$ and $\psi_{m, c}^{n}=0$, otherwise. $\varphi \in \mathbb{Z}^{K \times N}$ and $\psi \in \mathbb{Z}^{M \times N}$ denote the subchannel assignment variables in small-cell and UAV networks, respectively. $\mathbf{p} \in \mathbb{R}^{K \times N}$ and $\hat{\mathbf{p}} \in \mathbb{R}^{M \times N}$ are the collections of power allocation variables in small-cell and UAV networks. $P_{\max , c}^{\mathrm{SC}}$ and $P_{\max , c}^{\mathrm{UAV}}$ are the maximum total power of the SC or UAV base station at cell $c . R_{m, c}^{\min }$ represents the minimum data rate for UAU $m$ at cell $c . p_{c_{c}}$ and $\hat{p}_{c_{c}}$ are the circuitry power consumption for the $c$ th base station (either SC or UAV). Note that the objective functions (5a) and (5b) are not convex [17], and therefore, (5) is a non-convex optimization problem. One possible approach to solve an MOOP is the socalled weighted Tchebycheff method [13] which introduces an additional auxiliary optimization variable $\chi$ given as follows

$$
\begin{array}{ll} 
& \min _{\{\mathbf{p}, \hat{\mathbf{p}}, \boldsymbol{\varphi}, \boldsymbol{\psi}, \chi\}} \chi, \\
\text { s.t. } \quad & \alpha\left(\mathrm{EE}_{0, \mathrm{~S}-\mathrm{UE}}-\eta_{\mathrm{EE}_{\mathrm{S}-\mathrm{UE}}}\right)-\chi \leq 0, \\
& (1-\alpha)\left(\mathrm{EE}_{0, \mathrm{U}-\mathrm{UE}}-\eta_{\mathrm{EE}_{\mathrm{U}-\mathrm{UE}}}\right)-\chi \leq 0, \\
& (5 \mathrm{c})-(5 \mathrm{~g}) .
\end{array}
$$

where $\alpha$ and $(1-\alpha)$ denote the weight coefficients indicating the importance of the different objectives. $\mathrm{EE}_{0, \mathrm{~S} \text {-UE }}$ and $\mathrm{EE}_{0, \mathrm{U}-\mathrm{UE}}$ are the utopia values for energy efficiency of SC and UAV links, respectively. In the following, we propose a novel approach to solve (8) in which sub-channel assignment and power allocation are iteratively performed by first computing initial feasible solutions. Specifically, this iterative approach begins with computing an initial feasible solution for $\hat{\mathbf{p}}[\mathbf{0}]$, $\psi[0], \mathrm{p}[0]$ and $\varphi[0]$ where we investigate to find them in the following section.

\section{A. Initial Power Allocation and Subchannel Assignment}

To give the initial point for $\hat{\mathbf{p}}[\mathbf{0}]$, we use the following optimization for each cell $c \in \mathcal{C}$ as

$$
\begin{aligned}
& \max _{\hat{\mathbf{p}}[\mathbf{0}], \boldsymbol{\psi}[\mathbf{0}]} \frac{\sum_{m=1}^{m_{c}} \sum_{n=1}^{N} \ln \left(1+\frac{\hat{p}_{m, c}^{n}[0] g_{m, c}^{n}}{N_{0}}\right)}{\sum_{m=1}^{m_{c}} \sum_{n=1}^{N} \hat{p}_{m, c}^{n}[0]+\hat{p}_{c_{c}}}, \\
& \text { s.t. } \quad \sum_{m=1}^{m_{c}} \sum_{n=1}^{N} \hat{p}_{m, c}^{n}[0] \leq P_{\max , c}^{\mathrm{UAV}} \\
& \quad \sum_{n=1}^{N} \ln \left(1+\frac{\hat{p}_{m, c}^{n}[0] g_{m, c}^{n}}{N_{0}}\right) \geq R_{m, c}^{\min }, \forall m .
\end{aligned}
$$

We convert the optimization problem in (9) into an equivalent optimization problem using the concept of fractional programming [14]. In fractional programming where the objective function appears in the form of a ratio of two functions, the non-convex objective function is transformed to an equivalent convex function using the Dinkelback approach. So, from (9) we define a new objective function as

$$
\sum_{m=1}^{m_{c}} \sum_{n=1}^{N} \ln \left(1+\frac{\hat{p}_{m, c}^{n}[0] g_{m, c}^{n}}{N_{0}}\right)-b\left(\sum_{m=1}^{m_{c}} \sum_{n=1}^{N} \hat{p}_{m, c}^{n}[0]+\hat{p}_{c_{c}}\right) \text {, }
$$

where $b$ is a non-negative and auxiliary variable that is being updated at each iteration as the ratio of the nominator to the denominator of objective function. As shown in [14], the optimal power allocation of optimization problem (10) subject to (9b) and (9), i.e., $\hat{p}_{m, c}^{n^{*}}[0](b)$ at a certain value of the parameter $b$, denoted as $b^{*}$ is also the optimal solution to (9), i.e., $\hat{p}_{m, c}^{n^{*}}[0]$. By applying Lagrangian approach, we have

$$
\hat{p}_{m, c}^{n}[0]=\left[\frac{1+\varsigma_{m, c}}{\eta_{c}+b}-\frac{N_{0}}{g_{m, c}^{n}}\right]^{+},
$$

where $[.]^{+}$denotes $\max \{., 0\} . \eta_{c}$ and $\varsigma_{m, c}$ can be chosen such that

$$
\sum_{m=1}^{m_{c}} \sum_{n=1}^{N} \hat{p}_{m, c}^{n}[0]=P_{\max , c}^{\mathrm{UAV}},
$$




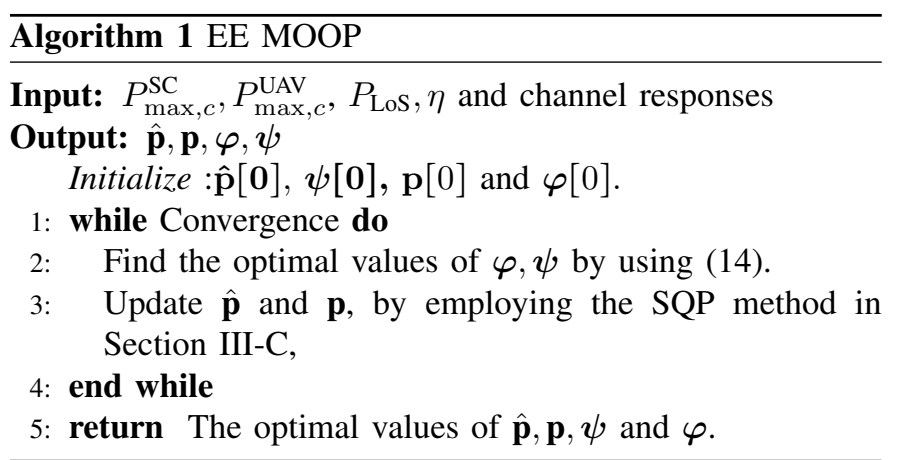

and

$$
\sum_{n=1}^{N} \ln \left(1+\frac{\hat{p}_{m, c}^{n}[0] g_{m, c}^{n}}{N_{0}}\right)=R_{m, c}^{\min }
$$

for each $m \in m_{c}$ and $c \in \mathcal{C}$. The optimal power allocation in (11) is obtained from the water-filling approach which can be selected as the feasible solution for transmit power of UAV base stations in each subchannel. The initial subchannel assignment for UAU $m, \boldsymbol{\psi}[\mathbf{0}]$, is determined with the highest channel-to-noise ratio on that subchannel. Applying similar approach, the initial values for $\mathrm{p}[0]$ and $\varphi[0]$ are derived.

\section{B. Subchannel Assignment for a Given Power}

For a given power in previous iteration, the optimal subchannel assignment for U-UE $m$ in cell $c$ over subchannel $n$ in current iteration $\tau$ is

$$
\psi_{m, c}^{n}[\tau]= \begin{cases}1, & \text { if } m=\arg \max _{m \in m_{c}} r_{m, c}^{n, \mathrm{U}-\mathrm{UE}}[\tau-1] \\ 0, & \text { otherwise }\end{cases}
$$

where $r_{m, c}^{n, \mathrm{U}-\mathrm{UE}}[\tau-1]=\ln \left(1+\Gamma_{m, c}^{n, \mathrm{U}-\mathrm{UE}}[\tau-1]\right)$. Similarly, the optimal subchannel assignment for S-UE $k, \varphi_{k, c}^{n}$, can be obtained.

\section{Power Allocation for a Given Subchannel}

After subchannel assignment at each iteration, to deal with power allocation we employ a technique known as sequential quadratic programming (SQP) which is recognized as a nonlinear programming algorithm for nonconvex constrained optimization problems. In terms of accuracy and efficiency, this method gives a better solution compared to the other nonlinear programming methods over a large number of test problems. At each iteration, a quadratic programming (QP) subproblem is generated by an approximation of the Hessian of the Lagrangian function. The solution of this QP provides a search direction for a line search procedure [15, Chapter
18]. In SQP technique, we should first form the Lagrangian function for (8), which yields

$$
\begin{aligned}
& L(\mathbf{p}, \hat{\mathbf{p}}, \lambda, \mu, \boldsymbol{\gamma}, \varrho, \boldsymbol{\xi})= \\
& \quad \chi+\mu\left(\alpha\left(\mathrm{EE}_{0, \mathrm{~S}-\mathrm{UE}}-\eta_{\mathrm{EE}_{\mathrm{S}-\mathrm{UE}}}\right)-\chi\right) \\
& \quad+\lambda\left((1-\alpha)\left(\mathrm{EE}_{0, \mathrm{U}-\mathrm{UE}}-\eta_{\mathrm{EE}_{\mathrm{U}-\mathrm{UE}}}\right)-\chi\right)+ \\
& \quad \sum_{c=1}^{C}\left[\gamma_{c}\left(\sum_{k=1}^{k_{c}} \sum_{n=1}^{N} p_{k, c}^{n}-P_{\max , c}^{\mathrm{SC}}\right)+\varrho_{c}\left(\sum_{m=1}^{m_{c}} \sum_{n=1}^{N} \hat{p}_{m, c}^{n}-P_{\max , c}^{\mathrm{UAV}}\right)\right] \\
& \quad+\sum_{c=1}^{C} \sum_{m=1}^{m_{c}} \xi_{m, c}\left(R_{m, c}^{\min }-\sum_{n=1}^{N} \ln \left(1+\Gamma_{m, c}^{n, \mathrm{U}-\mathrm{UE}}\right)\right)
\end{aligned}
$$

where $\mu, \lambda, \gamma=\left[\gamma_{c}\right]_{c \in \mathcal{C}}, \varrho=\left[\varrho_{c}\right]_{c \in \mathcal{C}}$ and $\boldsymbol{\xi}=\left[\xi_{m, c}\right]_{m \in m_{c}, c \in \mathcal{C}}$ are the non-negative Lagrangian multipliers. The solution $(\mathbf{p}, \hat{\mathbf{p}}, \lambda, \mu, \gamma, \varrho, \boldsymbol{\xi})$ can be updated as

$$
\left[\begin{array}{c}
\mathbf{p}^{l+1} \\
\hat{\mathbf{p}}^{l+1} \\
\lambda^{l+1} \\
\mu^{l+1} \\
\boldsymbol{\gamma}^{l+1} \\
\boldsymbol{\varrho}^{l+1} \\
\boldsymbol{\xi}^{l+1}
\end{array}\right]=\left[\begin{array}{c}
\mathbf{p}^{l}+s^{l} \nu^{l} \\
\hat{\mathbf{p}}^{l}+\hat{s}^{l} \hat{\nu}^{l} \\
\lambda^{l}+s_{\lambda}^{l} \nu_{\lambda}^{l} \\
\mu^{l}+s_{\mu}^{l} \nu_{\mu}^{l} \\
\boldsymbol{\gamma}^{l}+s_{\gamma}^{l} \nu_{\gamma}^{l} \\
\varrho^{l}+s_{\varrho}^{l} \nu_{\varrho}^{l} \\
\boldsymbol{\xi}^{l}+s_{\xi}^{l} \nu_{\xi}^{l}
\end{array}\right]
$$

where $s^{l}, \hat{s}^{l}, s_{\lambda}^{l}, s_{\mu}^{l}, s_{\gamma}^{l}, s_{\varrho}^{l}$ and $s_{\xi}^{l}$ are nonnegative step sizes at the $l$ th iteration to construct the new estimates. The vector $\boldsymbol{\nu}=\left[\begin{array}{lllllll}\nu & \hat{\nu} & \nu_{\lambda} & \nu_{\mu} & \nu_{\gamma} & \nu_{\varrho} & \nu_{\xi}\end{array}\right]^{T}$ can be given by solving the following quadratic programming problem

$$
\boldsymbol{\nu}=-\left[\begin{array}{cc}
\mathbf{H}(\mathbf{p}, \hat{\mathbf{p}}, \lambda, \mu, \boldsymbol{\gamma}, \boldsymbol{\varrho}, \boldsymbol{\xi}) & \mathbf{J}^{T}(\mathbf{p}, \hat{\mathbf{p}}) \\
\mathbf{J}(\mathbf{p}, \hat{\mathbf{p}}) & \mathbf{0}
\end{array}\right]^{-1} \times\left[\begin{array}{c}
g_{L} \\
\hat{g}_{L} \\
\mathbf{B}
\end{array}\right]
$$

where $g_{L}$ and $\hat{g}_{L}$ denote the gradient of the Lagrangian function (15) with respect to $p_{k, c}^{n}$ and $\hat{p}_{m, c}^{n}$, respectively. Furthermore, matrices $\mathbf{B}, \mathbf{J}(\mathbf{p}, \hat{\mathbf{p}})$ and $\mathbf{H}(\mathbf{p}, \hat{\mathbf{p}}, \lambda, \mu, \gamma, \boldsymbol{\varrho}, \boldsymbol{\xi})$ are defined as follows. The matrix $\mathbf{B}$ is denoted as

$$
\mathbf{B}=[a(\mathbf{p}, \hat{\mathbf{p}}) b(\mathbf{p}, \hat{\mathbf{p}}) c(\mathbf{p}, \hat{\mathbf{p}}) d(\mathbf{p}, \hat{\mathbf{p}}) e(\mathbf{p}, \hat{\mathbf{p}})]^{T}
$$

with the following elements

$$
\begin{aligned}
& a(\mathbf{p}, \hat{\mathbf{p}})=\alpha\left(\mathrm{EE}_{0, \mathrm{~S}-\mathrm{UE}}-\eta_{\mathrm{EE}_{\mathrm{S}-\mathrm{UE}}}\right)-\chi \\
& b(\mathbf{p}, \hat{\mathbf{p}})=(1-\alpha)\left(\mathrm{EE}_{0, \mathrm{U}-\mathrm{UE}}-\eta_{\mathrm{EE}_{\mathrm{U}-\mathrm{UE}}}\right)-\chi \\
& c(\mathbf{p}, \hat{\mathbf{p}})=\sum_{k=1}^{k_{c}} \sum_{n=1}^{N} p_{k, c}^{n}-P_{\mathrm{max}, c}^{\mathrm{SC}}, \\
& d(\mathbf{p}, \hat{\mathbf{p}})=\sum_{m=1}^{m_{c}} \sum_{n=1}^{N} \hat{p}_{m, c}^{n}-P_{\mathrm{max}, c}^{\mathrm{UAV}}, \\
& e(\mathbf{p}, \hat{\mathbf{p}})=R_{m, c}^{\min }-\sum_{n=1}^{N} \ln \left(1+\Gamma_{m, c}^{n, \mathrm{U}-\mathrm{UE}}\right) .
\end{aligned}
$$

Jacobian matrix of $\mathbf{B}$, denoted by matrix $\mathbf{J}$, consists of the partial derivatives of (19)-(23) with respect to $p_{k, c}^{n}$ and $\hat{p}_{m, c}^{n}$ 
according to

$$
\mathbf{J}(\mathbf{p}, \hat{\mathbf{p}})=\left[\begin{array}{ll}
\frac{\partial a(\mathbf{p}, \hat{\mathbf{p}})}{\partial \mathbf{p}} & \frac{\partial a(\mathbf{p}, \hat{\mathbf{p}})}{\partial \hat{\mathbf{p}}} \\
\frac{\partial b(\mathbf{p}, \hat{\mathbf{p}})}{\partial \mathbf{p}} & \frac{\partial b(\mathbf{p}, \hat{\mathbf{p}})}{\partial \hat{\mathbf{p}}} \\
\frac{\partial c(\mathbf{p}, \hat{\mathbf{p}})}{\partial \mathbf{p}} & \frac{\partial c(\mathbf{p}, \hat{\mathbf{p}})}{\partial \hat{\mathbf{p}}} \\
\frac{\partial d(\mathbf{p}, \hat{\mathbf{p}})}{\partial \mathbf{p}} & \frac{\partial d(\mathbf{p}, \hat{\mathbf{p}})}{\partial \hat{\mathbf{p}}} \\
\frac{\partial e(\mathbf{p}, \hat{\mathbf{p}})}{\partial \mathbf{p}} & \frac{\partial e(\hat{\mathbf{p}}, \hat{\mathbf{p}})}{\partial \hat{\mathbf{p}}}
\end{array}\right] .
$$

$\mathbf{H}(\mathbf{p}, \hat{\mathbf{p}}, \lambda, \mu, \gamma, \varrho, \boldsymbol{\xi})$ represents the Hessian matrix of Lagrangian function (15) which is

$$
\mathbf{H}(\mathbf{p}, \hat{\mathbf{p}}, \lambda, \mu, \boldsymbol{\gamma}, \boldsymbol{\varrho}, \boldsymbol{\xi})=\left[\begin{array}{cc}
\frac{\partial^{2} L}{\partial \mathbf{p}^{2}} & \frac{\partial^{2} L}{\partial \mathbf{p} \partial \hat{\mathbf{p}}} \\
\frac{\partial L}{\partial \hat{\mathbf{p}} \partial \mathbf{p}} & \frac{\partial L}{\partial \hat{\mathbf{p}}^{2}}
\end{array}\right] .
$$

In SQP method, a merit function is employed to decide whether an iteration can be accepted or not. Several merit functions have been used in SQP methods, however, augmented Lagrangians and non-smooth penalty functions [15] are the most popular merit functions. By choosing $\omega(\mathbf{p}, \hat{\mathbf{p}})$ as a merit function and a value for $\mathbf{s}^{l}=$ $\left[\begin{array}{ccccccc}s^{l} & \hat{s}^{l} & s_{\lambda}^{l} & s_{\mu}^{l} & s_{\gamma}^{l} & s_{\varrho}^{l} & s_{\xi}^{l}\end{array}\right]^{T}$, we know that when $\omega\left(\mathbf{p}^{l}+\mathbf{s}^{l} \nu^{l}, \hat{\mathbf{p}}^{l}+\mathbf{s} \nu\right) \leq \omega(\mathbf{p}, \hat{\mathbf{p}})$, the current iteration results will be accepted as well. The overall procedure to solve (5) is summarized in Algorithm 1.

\section{Complexity Analysis}

In this section, we briefly investigate the computational complexity of SQP method. The SQP method is implemented in a variety of simulation programs where the objective function is twice differentiable. Taking a look at (15), we conclude that the computational complexity of Lagrangian function is $\mathcal{O}\left(C \times\left\{\max _{c} m_{c}\right\} \times N\right)+\mathcal{O}\left(C \times\left\{\max _{c} k_{c}\right\} \times N\right)$. Furthermore, to evaluate (17), we need to compute the inner matrices $\mathbf{H}, \mathbf{J}$ and $\mathbf{B}$ in which the complexity grows with $\mathcal{O}\left(C \times\left\{\max _{c} m_{c}\right\} \times N\right)+\mathcal{O}\left(C \times\left\{\max _{c} k_{c}\right\} \times N\right)$.

\section{NUMERICAL RESUlTS}

In this section, we present the numerical results for a tradeoff analysis between the achievable EE of UAV and SC networks in the OFDMA-based small-cell and UAV network. The users and base stations' locations are generated in 2D plane according to Poisson point process (PPP) distribution. For simplicity, we assume that the UAV base stations are in a fixed position during the signal transmission. The simulation parameters are given in Table. I.

Fig. 2 shows the optimization of the EE of UAV and SC networks in terms of the weight coefficient $(\alpha)$ with $p_{c_{c}}=$ $\hat{p}_{c_{c}}=-7 \mathrm{~dB}$. The results reveal a tradeoff between the EEs of these two networks. As we observe, by increasing $\alpha$, the EE of S-UEs increases until some saturation value is reached, on the other hand, the EE of UAV links decreases as well.
TABLE I: Simulation parameters

\begin{tabular}{ll}
\hline Parameter & Value \\
\hline Number of U-UEs $(M)$ & 4 \\
Number of S-UEs $(K)$ & 4 \\
Number of subbands $(N)$ & 4 \\
Minimum data rate $\left(R_{m, c}^{\min }\right)$ & $0.3 \mathrm{nat} / \mathrm{s} / \mathrm{Hz}$ \\
Maximum power of SC $\left(P_{\max , c}^{\mathrm{SC}}\right)$, normalized & $10 \mathrm{~dB}$ \\
Maximum power of UAV $\left(P_{\max , c}^{\mathrm{UAV}}\right)$, normalized & $15 \mathrm{~dB}$ \\
UAV channel excess attenuation $(\eta)$ & $-20 \mathrm{~dB}$ \\
UAV channel parameter $\left(\kappa_{1}\right)$ & 0.136 \\
UAV channel parameter $\left(\kappa_{2}\right)$ & 11.95 \\
Noise variance $\left(N_{0}\right)$ & 1 \\
\hline
\end{tabular}

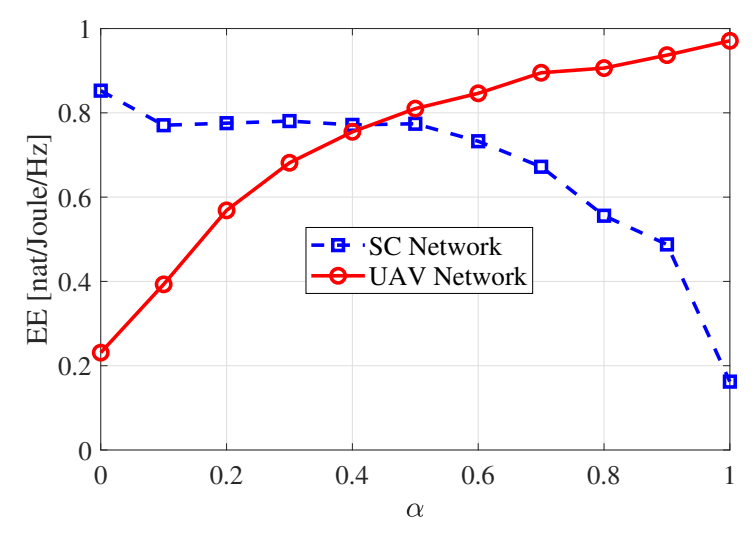

Fig. 2: Maximum EE of UAV and SC networks vs. $\alpha$.

To demonstrate the advantage of MOOP in (5), we here formulate the following SOOP where the EE of only the S-UEs is maximized subject to the same constraints, i.e., the objective (5b) is removed from MOOP in (5), simplifying MOOP into a SOOP as

$$
\begin{aligned}
& \max _{\{\mathbf{p}, \hat{\mathbf{p}}, \boldsymbol{\varphi}\}} \eta_{\mathrm{EE}_{\mathrm{S}-\mathrm{UE}}} \\
& \text { s.t. } \quad(5 \mathrm{c}),(5 \mathrm{e}) \text { and }(5 \mathrm{f}) .
\end{aligned}
$$

We take the results of solving SOOP in (24) as a benchmark and compare them with the results of MOOP in Fig. 3 where the total EE of both S-UEs and U-UEs (i.e., $\left.\frac{\text { Total rate of S-UEs and U-UEs }}{\text { Total power of BSs+Circuitry power consumption }}\right)$ versus $\alpha$ for MOOP in (5) and SOOP in (24) is illustrated. Fig. 3 reveals that the EE of both S-UEs and U-UEs in MOOP is more than EE of both S-UEs and U-UEs in SOOP. We can conclude that solving MOOP results in higher EE in the network particularly when we consider both objective functions with the same weight coefficients $\alpha=0.5$. As it is inferred, the maximum value for $\mathrm{EE}$ is obtained $\mathrm{EE}_{\mathrm{opt}}^{\mathrm{total}}=1.4$ nat $/ \mathrm{Joule} / \mathrm{Hz}$ at about $\alpha \approx 0.5$ while as we compare it with the intersection point in which the two EE curves in Fig. 2 have the same value of $\mathrm{EE}_{\mathrm{opt}}=\mathrm{EE}_{\mathrm{opt}}^{\mathrm{SC}}+\mathrm{EE}_{\mathrm{opt}}^{\mathrm{UAV}}=1.8$ nat $/ \mathrm{Joule} / \mathrm{Hz}$ at $\alpha \approx 0.45$, we see that the total EE of the network is reached at about the same weight coefficient of maximizing two individual EEs of UAVs and SCs. 


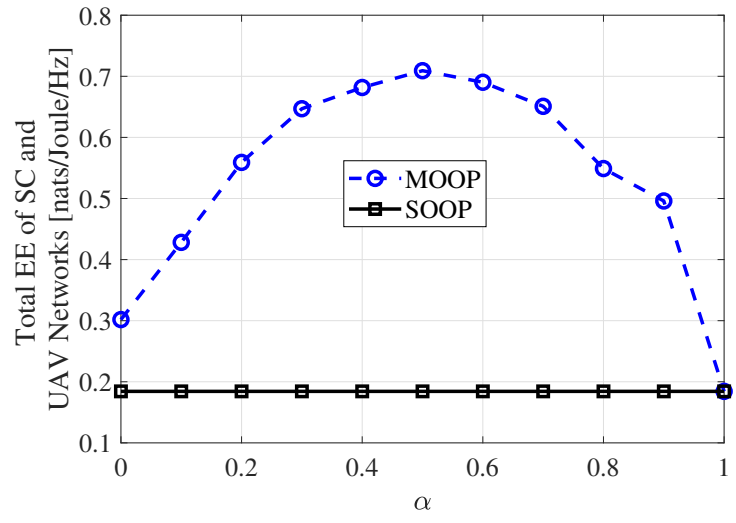

Fig. 3: Comparing MOOP with SOOP.

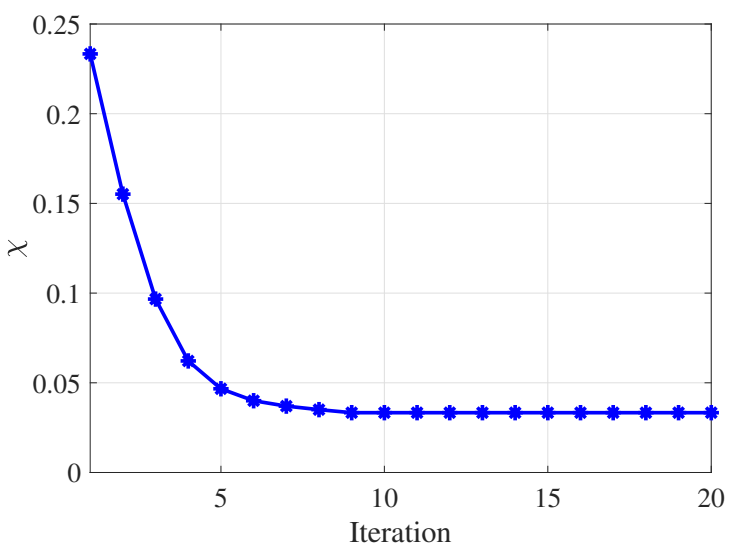

Fig. 4: The iterative minimization of Tchebyshev variable for solving MOOP by using SQP method.

Next, in order to illustrate the convergence speed of the Tchebyshev algorithm, we demonstrate the speed of decreasing $\chi$ in terms of the number of iterations in Fig. 4. As we see, the algorithm converges quickly at 5-6 iterations for $\chi \approx 0.04$. We conclude this section by computing the order of convergence using the results given in Fig. 4. In order to do that, we note that

$$
q \approx \frac{\log \left(\left|\frac{\chi_{k+1}-\chi_{k}}{\chi_{k}-\chi_{k-1}}\right|\right)}{\log \left(\left|\frac{\chi_{k}-\chi_{k-1}}{\chi_{k-1}-\chi_{k-2}}\right|\right)},
$$

hence, evaluating the order of convergence from the Fig. 4 yields $q \approx 2$ which confirms quadratic convergence of SQP method.

\section{CONCLUSION}

In this paper, we considered a downlink multi-cell OFDMAbased small-cell UAV network where the UAV-BSs are transmitting along with SCs in the same frequency band. First, we have formulated a MOOP to jointly maximize the EE of SUEs and U-UEs where a minimum data rate constraint for UAV users is also considered to preserve the UAV-UEs' QoS. In contrast to the most prior papers that only maximize the EE of SCs, we introduced a novel tradeoff between the EE of
UAV and SC networks by formulating the MOOP. Then, to solve the proposed optimization problems, we have employed a general mathematical method know as SQP that is widely used to solve non-convex problems. In numerical results, we have shown that there is a fundamental tradeoff between the EE of SC and UAV networks and the evaluations indicate the superiority of proposed MOOP over SOOP in terms of total EE of the network. We have also observed that the SQP algorithm converges quickly and it is best suitable to solve the presented MOOP.

\section{REFERENCES}

1] M. Mozaffari, W. Saad, M. Bennis, Y. -H. Nam and M. Debbah, "A Tutorial on UAVs for Wireless Networks: Applications, Challenges, and Open Problems, "in IEEE Communications Surveys \& Tutorials, vol. 21, no. 3, pp. 2334-2360, 2019.

[2] Y. Zeng, R. Zhang and T. J. Lim, "Wireless communications with unmanned aerial vehicles: opportunities and challenges,"in IEEE Communications Magazine, vol. 54, no. 5, pp. 36-42, May 2016.

[3] J. G. Andrews, "Seven ways that HetNets are a cellular paradigm shift,"in IEEE Communications Magazine, vol. 51, no. 3, pp. 136-144, March 2013.

[4] M. Jaber, M. A. Imran, R. Tafazolli and A. Tukmanov, "5G Backhaul Challenges and Emerging Research Directions: A Survey,"in IEEE Access, vol. 4, pp. 1743-1766, 2016.

[5] M. Mozaffari, W. Saad, M. Bennis and M. Debbah, "Unmanned Aerial Vehicle With Underlaid Device-to-Device Communications: Performance and Tradeoffs, "IEEE Transactions on Wireless Communications, vol. 15 , no. 6, pp. 3949-3963, June 2016.

[6] A. Merwaday and I. Guvenc, "UAV assisted heterogeneous networks for public safety communications,"2015 IEEE Wireless Communications and Networking Conference Workshops (WCNCW), New Orleans, LA 2015, pp. 329-334.

[7] V. Sharma, M. Bennis and R. Kumar, "UAV-Assisted Heterogeneous Networks for Capacity Enhancement,"in IEEE Communications Letters, vol. 20, no. 6, pp. 1207-1210, June 2016.

[8] J.-H. Cho, Y. Wang, I.-R. Chen, K. S. Chan, and A. Swami, "A survey on modeling and optimizing multi-objective systems," IEEE Communications Surveys and Tutorials, vol. 19, no. 3, pp. 1867-1901, 3rd Quart., 2017.

[9] J. Rubio, A. Pascual-Iserte, D. P. Palomar and A. Goldsmith, "Joint Optimization of Power and Data Transfer in Multiuser MIMO Systems," IEEE Transactions on Signal Processing, vol. 65, no. 1, pp. 212-227, Jan. 2017.

[10] M. R. Mili, L. Musavian, K. A. Hamdi and F. Marvasti, "How to Increase Energy Efficiency in Cognitive Radio Networks," IEEE Transactions on Communications, vol. 64, no. 5, pp. 1829-1843, May 2016.

[11] H. Tran, G. Kaddoum and K. T. Truong, "Resource Allocation in SWIPT Networks Under a Nonlinear Energy Harvesting Model: Power Efficiency, User Fairness, and Channel Nonreciprocity," IEEE Transactions on Vehicular Technology, vol. 67, no. 9, pp. 8466-8480, Sep. 2018.

[12] R. Hashemi, H. Beyranvand, M. R. Mili, A. Khalili, H. Tabassum, and D. W. K. Ng, "Energy efficiency maximization in the uplink delta-OMA networks," Feb., 2021. [Online]. Available: http://arxiv.org/abs/2102.13359.

[13] K. Miettinen, Nonlinear Multiobjective Optimization, Springer, vol. 12, 2012.

[14] W. Dinkelbach, "On nonlinear fractional programming," Management Science, vol. 13, no. 7, pp. 492-498, Mar. 1967.

[15] J. Nocedal and S. J. Wright, Numerical Optimization, Second ed. Springer-Verlag, New York Inc, 2006.

[16] A. Al-Hourani, S. Kandeepan and S. Lardner, "Optimal LAP Altitude for Maximum Coverage,"in IEEE Wireless Communications Letters, vol. 3, no. 6, pp. 569-572, Dec. 2014.

[17] S. Boyd and L. Vandenberghe, Convex optimization. Cambridge university press, 2004. 